\title{
Gastric mucosa in patients with portal hypertension: prevalence of capillary dilatation and Campylobacter pylori
}

\author{
P N FOSTER, J I WYATT, D W BULLIMORE, M S LOSOWSKY \\ From the Departments of Medicine and Pathology, St James's University Hospital, Leeds
}

SUMMARY To determine whether the "congestive" gastropathy associated with portal hypertension showed distinctive histological features independent of inflammatory gastritis, endoscopic biopsy specimens of gastric mucosa from 23 patients with portal hypertension and 25 patients with non-ulcer dyspepsia were examined. Active chronic gastritis associated with Campylobacter pylori was found in three patients with portal hypertension compared with 13 patients with non-ulcer dyspepsia. The changes of reflux gastritis were seen in nine patients with portal hypertension compared with three patients with non-ulcer dyspepsia. Mucosal capillary dilatation, assessed on sections stained for factor VIII related antigen, a specific marker for endothelial cells, was significantly greater in biopsy specimens from patients with portal hypertension but this difference was not apparent on sections stained conventionally. The degree of capillary dilatation was unrelated to the presence of histological gastritis.

These observations support the view that portal hypertension is associated with a distinctive gastropathy characterised by prominence and dilatation of mucosal capillaries.

Gastric mucosal lesions are common in portal hypertension and may be an important cause of bleeding. ${ }^{1}$ Several studies have reported the endoscopic features of the gastric mucosa in patients with portal hypertension, ${ }^{23}$ and it has been suggested that there is a particular gastropathy associated with portal hypertension which is characterised histologically by vascular dilatation in the mucosa and submucosa in excess of that expected with the degree and activity of any concomitant gastritis. ${ }^{4}$ The original description of these histological features was based on examination of material obtained at necropsy, operation, and endoscopy but the value of specimens taken by endoscope in diagnosing this "congestive" gastropathy has been questioned recently." Because endoscopy offers the simplest method of sampling the gastric mucosa to establish the diagnosis, we undertook a study to determine whether the congestive gastropathy of portal hypertension shows distinctive features, independent of inflammatory gastritis, in endoscopically obtained biopsy specimens from patients with portal hypertension and patients with non-ulcer dyspepsia. In addition to routine staining of

Accepted for publication 16 March 1989 specimens with haematoxylin and eosin, we used an immunoperoxidase stain for factor VIII related antigen, a specific marker for endothelium, to highlight the vessel walls.

\section{Patients and methods}

Twenty three patients (13 men, 10 women) aged 18 to 77 years (mean 48.1) undergoing injection sclerotherapy of oesophageal varices were studied. The cause of the portal hypertension was chronic liver disease in 19 patients, extrahepatic portal vein thrombosis in three, and idiopathic non-cirrhotic portal hypertension in one (table 1). Four patients were

Table 1 Aetiology of chronic liver disease in 19 patients

\begin{tabular}{ll}
\hline Condition & No of patients \\
\hline Alcoholic cirrhosis & 4 \\
Primary biliary cirrhosis & 3 \\
Secondary biliary cirrhosis & 2 \\
Hepatitis B cirrhosis & 1 \\
Autoimmune chronic active hepatitis & 2 \\
Alpha-1-anti-trypsin deficiency & 1 \\
Granulomatous hepatitis & 1 \\
Cryptogenic cirrhosis & 5 \\
\hline
\end{tabular}




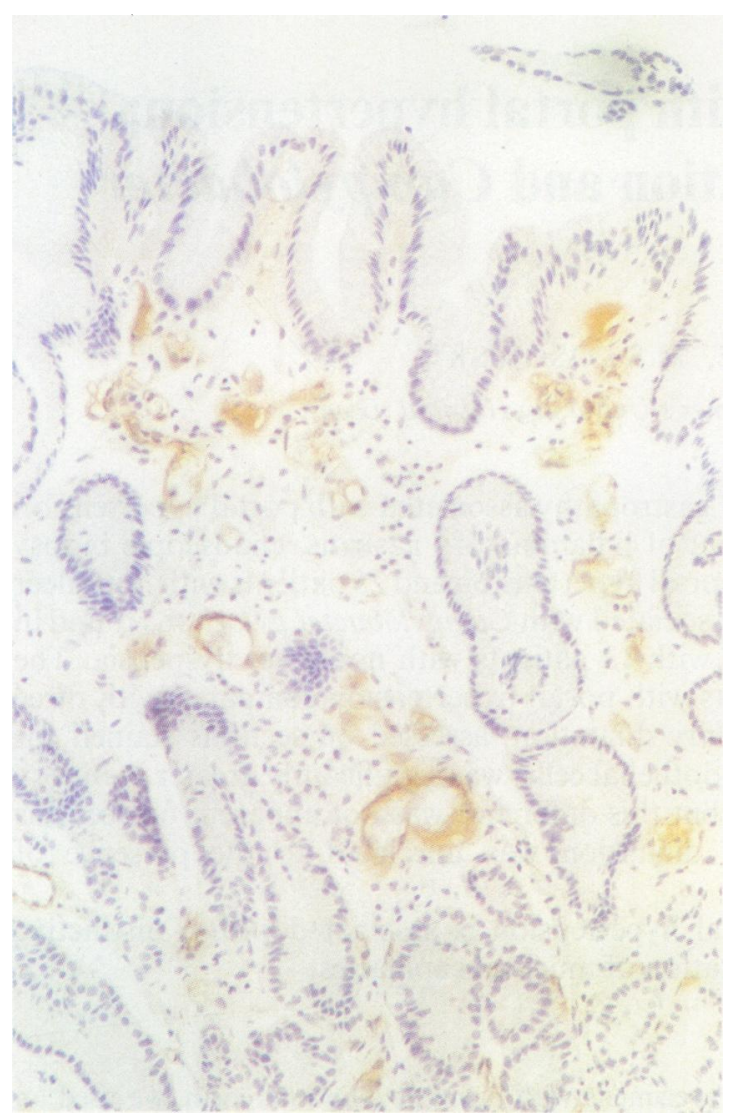

Figure Gastric antral mucosa from a patient with portal hypertension immunostained for factor VIII related antigen, showing prominent, ectatic capillaries in the superficial lamina popria, corresponding to grade 3 dilatation.

attending for their first session of sclerotherapy; the remainder had undergone between one and nine sessions (median four), and in 12 patients the varices had been obliterated. Twenty five patients with symptoms of dyspepsia but no macroscopic lesion at endoscopy served as controls. Forty five gastric mucosal biopsy specimens ( 33 antral; 12 body) from the patients with portal hypertension and 44 from the controls ( 26 antral; 18 body) were obtained endoscopically (Olympus Gifit 10/GIFQ10).

Gastritis was graded morphologically according to Whitehead's criteria. ${ }^{6}$ The degree of vascular dilatation, congestion (distension of mucosal vessels by blood), and fresh mucosal hemorrhage were each graded using a scale 0 to 3 on sections stained with haematoxylin and eosin. Mucosal vascularity was also assessed and graded similarly on sections stained for factor VIII related antigen (Dako Immunoglobulins), a specific marker for endothelium, ${ }^{7}$ by the indirect immunoperoxidase technique. Mucosal haemosiderin was identified by Perls's stain and the presence of $C$ pylori was detected on sections prepared with a modified Giemsa stain. ${ }^{8}$ The histological features were assessed blindly by one of us (JW).

RIDIT analysis ${ }^{9}$ of the data was performed.

\section{Results}

Eleven of the 23 patients with portal hypertension had macrospically abnormal mucosa ranging from hyperaemia to florid gastritis with erosions. There was no histological inflammation in the mucosa from five patients with portal hypertension and nine with nonulcer dyspepsia. Of the remaining 18 patients with portal hypertension, three had active chronic gastritis with $C$ pylori colonisation, five had inactive chronic atrophic gastritis (one with $C$ pylori), and biopsy specimens from nine showed histological features of "reflux gastritis". ${ }^{10}$ In the control group of patients 13 had Campylobacter associated active chronic gastritis and three showed features suggestive of reflux gastritis. All 12 patients with reflux gastritis were Campylobacter negative.

Dilatation of the capillaries, particularly in the superficial foveolar region of the mucosa (figure), was significantly greater in sections stained for factor VIII related antigen from patients with portal hypertension compared with controls ( $p<0.0001$; table 2$)$. This difference was not apparent in sections stained with haematoxylin and eosin (table 2). The degree of vascular dilatation was seen to be independent of the presence of inflammatory gastritis in patients with portal hypertension, although mild changes were associated with gastritis in the control group (table 3 ), and was unrelated to the macroscopic appearance of the gastric mucosa at endoscopy, the number of scleropathy sessions performed, or obliteration of the oesophageal varices.

There was no difference in the degree of congestion or fresh mucosal haemorrhage into the lamina propria between the two groups. The Perls's reaction showed stainable iron in the mucosa of eight patients with portal hypertension and in eight of the controls. This

Table 2 Degree of capillary dilatation using stain for factor VIII related antigen and haemotoxylin and eosin

\begin{tabular}{lllllll}
\hline & \multicolumn{2}{l}{ Portal hypertension } & & \multicolumn{2}{l}{ Non-ulcer dsypepsia } \\
\cline { 2 - 3 } \cline { 5 - 6 } Grade & FVIII RAg* & H and E† & & FVIII RAg* & H and E† \\
\hline 0 & 3 & 15 & & 23 & 21 \\
1 & 17 & 21 & & 18 & 17 \\
2 & 18 & 9 & & 3 & 5 \\
3 & 7 & 0 & 0 & 1 \\
\hline
\end{tabular}

*p $<0.0001$.

$+\mathrm{p}=0 \cdot 2$. 
Table 3 Degree of capillary dilatation using stain for factor VIII related antigen related to presence or absence of inflammatory gastritis

\begin{tabular}{|c|c|c|c|c|}
\hline & \multicolumn{4}{|c|}{ Grade } \\
\hline & 0 & 1 & 2 & 3 \\
\hline $\begin{array}{l}\text { Portal hypertension: } \\
\text { Present } \\
\text { Absent }\end{array}$ & $\begin{array}{l}2 \\
1\end{array}$ & $\begin{array}{r}4 \\
13\end{array}$ & $\begin{array}{l}9 \\
9\end{array}$ & $\begin{array}{l}3 \\
4 \\
\text { (NS) }\end{array}$ \\
\hline $\begin{array}{l}\text { Non-ulcer dyspepsia: } \\
\text { Present } \\
\text { Absent }\end{array}$ & $\begin{array}{r}6 \\
17\end{array}$ & $\begin{array}{r}15 \\
3\end{array}$ & $\begin{array}{l}1 \\
2\end{array}$ & $\begin{array}{l}0 \\
0 \\
(p=0.01)\end{array}$ \\
\hline
\end{tabular}

was usually very small in amount but differed in distribution between the two groups, being present in macrophages in the lamina propria in all eight cases associated with portal hypertension, while in the controls the iron was within epithe:ial cells at the deep aspect of the glands in five cases and in mucosal macrophages in three.

\section{Discussion}

Our observations support the view that portal hypertension is associated with a distinctive gastropathy characterised by prominence and dilatation of mucosal capillaries. ${ }^{4}$ Like Corbishley et $a l^{5}{ }^{5}$ who concluded that capillary dilatation was a non-specific finding, we found that these features were not readily apparent in endoscopic gastric biopsy specimens stained conventionally with haematoxylin and eosin, but the use of an immunoperoxidase stain for factor VIII related antigen, a specific marker for endothelium, to highlight the vessel walls made assessment of dilatation easier, allowing attention to be focused on the mucosal capillaries independently of other abnormalities.

Congestion is often apparent in gastric mucosa showing the inflammatory infiltrate of chronic gastritis, but we found that the prominence and ectasia of mucosal capillaries in portal hypertension develops independently of gastric inflammation. The histological features characterising reflux gastritis (foveolar hyperplasia, congestion and oedema, with paucity of inflammatory cells) were apparent in a high proportion of the cases with portal hypertension. It is not clear whether these appearances were due to a genuine reflux gastritis or whether the changed mucosal haemodynamics in portal hypertension produce a histological picture which mimics reflux gastritis.

Colonisation by $C$ pylori was detected in four $(17 \%)$ patients with portal hypertension which is fewer than would be expected in the age-matched general population. The prevalence of $C$ pylori associated gastritis increases with age and in our area approaches $50 \%$ of healthy subjects aged over 50 years. " This observation suggests that the gastric mucosa of patients with portal hypertension may be an inhospitable environment for C pylori.

The exact mechanism underlying the vascular abnormalities of congestive gastropathy is not clear. The raised portal venous pressure is probably important, but Quintero et al $^{3}$ found no correlation between capillary ectasia and portal pressure. McCormack et $\mathrm{al}^{4}$ suggested that changes in local blood flow consequent on sclerotherapy might be important. We observed no correlation between the degree of vascular dilatation and the number of sclerotherapy sessions or obliteration of the oesophageal varices. Finally, hypergastrinaemia and low pepsinogen concentrations have been found in patients with cirrhosis and vascular ectasia, ${ }^{3}$ but the clinical importance of these findings is uncertain.

The importance of the entity lies in the fact that the gastropathy seems to be a major source of haemorrhage in patients with portal hypertension and that there is some evidence that chronic $\beta$ blockade may reduce the risk of bleeding from this lesion. ${ }^{12}$

\section{References}

1 Waldram R, Davis M, Nunnerly H, Williams R. Emergency endoscopy after gastrointestinal haemorrhage in $\mathbf{5 0}$ patients with portal hypertension. Br Med J 1974;4:94-6.

2 Papazain A, Braillon A, Dupas JL, Sevenet F, Capron JP. Portal hypertensive gastric mucosa: an endoscopic study. Gut 1986; 27:1199-203.

3 Quintero E, Pique JM, Bombi JM, et al. Gastric mucosal vascular ectasias causing bleeding in cirrhosis. Gastroenterology 1987; 93:1054-61.

4 McCormack TT, Sims J,Eyre-Brook I, Goepel J, Johnson AG, Triger DR. Gastric lesions in portal hypertension: inflammatory gastritis or congestive gastropathy? Gut 1985;26: 1226-32.

5 Corbishley CM, Saverymuttu SH, Maxwell JD. Use of endoscopic biopsy for diagnosing congestive gastropathy. J Clin Pathol 1988;41:1187-90.

6 Whithead R, Trulove SC, Gear MWL. The histological diagnosis of chronic gastritis in fibreoptic gastroscope biopsy specimens. $J$ Clin Pathol 1972;25:1-11.

7 Mukai K, Rosai J, Burgdorf WHC. Localization of factor VIII related antigen in vascular endothelial cells using an immunoperoxidase method. Am J Clin Pathol 1980;4:273-6.

8 Gray SF, Wyatt JI, Rathbone BJ. Simplified techniques for identifying Campylobacter pyloridis. J Clin Pathol 1986;39:1279-80.

9 Bullimore D. RIDIT analysis for qualitative and subjective clinical data. Aliment Pharmacol Ther 1988;2:43-5.

10 Dixon MF, O'Conner HJ, Axon ATR, King RFJG, Johnston D. Reflux gastritis: a distinct histopathological entity? $J$ Clin Pathol 1986;39:524-30.

11 Wyatt JI, Rathbone BJ, Heatley RV, Losowsky MS. Campylobacter pylori and history of dyspepsia in healthy blood donors. Gut 1988;29:A706-7.

12 Hosking SW, Kennedy HJ, Seddon I, Triger DR. The role of propranolol in congestive gastropathy of portal hypertension. Hepatology 1987;7:437-41.

Requests for reprints to: Dr P N Foster, Department of Medicine, St James's Hospital, Leeds LS9 7TF, England. 